\title{
Dilaton chiral perturbation theory and applications
}

\author{
Maarten Golterman ${ }^{1, *}$ and Yigal Shamir ${ }^{2, * *}$ \\ ${ }^{1}$ Department of Physics and Astronomy, San Francisco State University, \\ San Francisco, CA 94132, USA \\ ${ }^{2}$ Raymond and Beverly Sackler School of Physics and Astronomy, \\ Tel Aviv University, 69978, Tel Aviv, Israel
}

\begin{abstract}
We review dilaton chiral perturbation theory (dChPT), the effective low-energy theory for the light sector of near-conformal, confining theories. dChPT provides a systematic expansion in both the fermion mass and the distance to the conformal window. It accounts for the pions and the light scalar, the approximate Nambu-Goldstone bosons for chiral and scale symmetry, respectively. A unique feature of dChPT is the existence of a large-mass regime in which the theory exhibits approximate hyperscaling, while the expansion nevertheless remains systematic. We discuss applications to lattice data, presenting successes as well as directions for future work.
\end{abstract}

\section{Introduction}

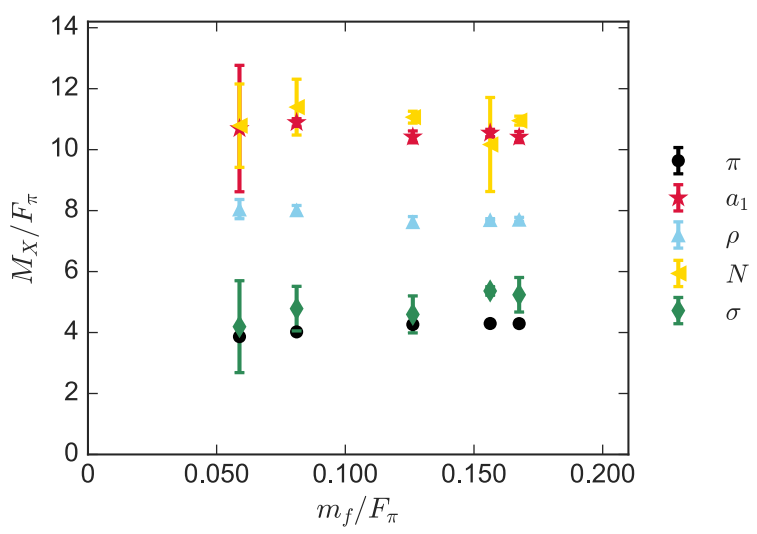

Figure 1. Hadron masses as a function of fermion mass, all in units of $F_{\pi}$, in the $S U(3)$ gauge theory with 8 fundamental flavors, from Ref. [2].

Let us start with a look at spectral data for the SU(3) gauge theory with $N_{f}=8$ fundamental fermions obtained by the LSD collaboration [1, 2], shown in Fig. 1. In comparison

\footnotetext{
*e-mail: maarten@sfsu.edu

**e-mail: shamir@tauex.tau.ac.il
} 
with QCD, several salient differences stand out: First, we note the presence of a stable $0^{++}$ state, denoted by the points labeled $\sigma$, with a mass virtually degenerate with the pions, over this mass range. Second, within errors, the spectrum shows hyperscaling: hadronic mass ratios are essentially independent of the fermion mass. In addition, pion "taste splittings" associated with the use of staggered fermions in all these simulations, i.e., non-degeneracies in the pion spectrum due to scaling violations, behave very differently as a function of the fermion mass in comparison with QCD (not shown in the figure). Similar results have been found in Ref. [3] by the LatKMI collaboration for the same theory, and in the SU(3) theory with two sextet fermions by the LatHC collaboration [4, 5], or with four light and six [6] or eight [7] heavy fundamental fermions. In this talk, we will discuss to what extent these data are decribed by tree-level dilaton Chiral Perturbation Theory (dChPT).

After a brief review of dChPT and its lowest-order lagrangian, we will explain the existence of a "large-mass" regime in which dChPT predicts approximate hyperscaling, as seen in Fig. 1. Going beyond hyperscaling, we will then apply dChPT to fit the LSD and LatKMI data, including the measured staggered taste splittings. We conclude with a brief discussion of what might be next.

\section{Lowest-order dChPT}

Dilaton ChPT is based on the following set of assumptions [8, 9]:

- Every gauge theory below the conformal sill contains Nambu-Goldstone bosons, or "pions," associated with the spontaneous breaking of chiral symmetry. The pions become massless in the limit $m \rightarrow 0$, where $m$ is the (degenerate) fermion mass.

- Scale invariance gets restored in the infrared as we approach the conformal window, with the trace anomaly being proportional to the distance to the conformal window. In the Veneziano limit $N_{f} \rightarrow \infty, N_{c} \rightarrow \infty$, with $n_{f}=N_{f} / N_{c}$ fixed, this happens when $n_{f}$ approaches a critical value $n_{f}^{*}$ from below. The difference $n_{f}-n_{f}^{*}$ is a new small parameter.

- The theory contains a dilaton, i.e., a Nambu-Goldstone boson associated with scale symmetry breaking, which becomes massless in the double limit $n_{f}-n_{f}^{*} \rightarrow 0$ and $m \rightarrow 0$.

- In addition, some technical assumptions on the dilaton potential are needed, see Ref. [10]. With these assumptions, one can prove that a systematic power counting in the small parameters

$$
p^{2} \sim m \sim n_{f}-n_{f}^{*} \sim 1 / N_{c}
$$

exists, and construct the lowest-order $\left(O\left(p^{2}\right)\right)$ lagrangian,

$$
\begin{aligned}
\mathcal{L}= & \frac{1}{4} f_{\pi}^{2} e^{2 \tau} \operatorname{tr}\left(\partial_{\mu} \Sigma^{\dagger} \partial_{\mu} \Sigma\right)+\frac{1}{2} f_{\tau}^{2} e^{2 \tau} \partial_{\mu} \tau \partial_{\mu} \tau \\
& -\frac{1}{2} f_{\pi}^{2} B_{\pi} m e^{\left(3-\gamma_{*}\right) \tau} \operatorname{tr}\left(\Sigma+\Sigma^{\dagger}\right)+f_{\tau}^{2} B_{\tau} e^{4 \tau} c_{1}\left(\tau-\frac{1}{4}\right)
\end{aligned}
$$

Here $\Sigma=\exp \left(2 i \pi / f_{\pi}\right)$ is the non-linear pion field and $\tau$ is the dilaton field. $c_{1}$ is a parameter proportional to $n_{f}-n_{f}^{*}$, of order $p^{2}$ in our power counting. At leading order, five low-energy constants (LECs) appear: $f_{\pi, \tau}, B_{\pi, \tau}$, and $\gamma_{*}$, the mass anomalous dimension at the infra-red fixed point at the conformal sill. For a detailed discussion of the lagrangian and the power counting, see Ref. [8]. We have used the freedom to shift the field $\tau$ such that $v(m)=\langle\tau\rangle$ vanishes for $m=0$, at this order.

The saddle point equation for the classical solution $v(m)$ is

$$
\frac{m}{c_{1} \mathcal{M}}=v(m) e^{\left(1+\gamma_{*}\right) v(m)}, \quad \mathcal{M} \equiv \frac{4 f_{\tau}^{2} B_{\tau}}{f_{\pi}^{2} B_{\pi} N_{f}\left(3-\gamma_{*}\right)} .
$$


The tree-level pion and dilaton masses, and their decay constants, are, using Eq. (3),

$$
\begin{aligned}
M_{\pi}^{2} & =2 B_{\pi} m e^{\left(1-\gamma_{*}\right) v(m)}=2 B_{\pi} c_{1} \mathcal{M} v(m) e^{2 v(m)}, \\
M_{\tau}^{2} & =4 B_{\tau} c_{1} e^{2 v(m)}\left(1+\left(1+\gamma_{*}\right) v(m)\right), \\
F_{\pi, \tau} & =f_{\pi, \tau} e^{v(m)} .
\end{aligned}
$$

Unlike in ordinary ChPT, here the tree-level decay constant(s) depend on the fermion mass via the factor $e^{v(m)}$.

\section{The small- and large-mass regimes}

An important observation is that in our power counting the ratio $\frac{m}{c_{1} \mathcal{M}}$ is parametrically $O(1)$. But this ratio can still be small, corresponding to the small-mass regime, or large, corresponding to the large-mass regime.

In the small-mass regime, $\frac{m}{c_{1} \mathcal{M}} \ll 1$, and $v \propto m$ while $e^{v}=1+O(m)$. One has $M_{\pi}^{2}=$ $2 B_{\pi} m \ll M_{\tau}^{2}=4 B_{\tau} c_{1} \propto\left|n_{f}-n_{f}^{*}\right|$. Even though, relative to the pions, the dilaton decouples, its mass still remains parameterically smaller than that of all other hadrons. For energies below the scale set by the dilaton mass the physics of pions only is described by standard ChPT.

The large-mass regime, $\frac{m}{c_{1} \mathcal{M}} \gg 1$, is more interesting [10]. In Eq. (3) the exponential dominates, and this equation has the approximate solution

$$
e^{v(m)} \sim\left(\frac{m}{c_{1} \mathcal{M}}\right)^{1 / 1+\gamma_{*}}
$$

In turn, this implies that Eq. (4) predicts approximate hyperscaling:

$$
M_{\pi} \sim M_{\tau} \sim F_{\pi} \sim F_{\tau} \sim M_{\mathrm{h}} \sim m^{1 / 1+\gamma_{*}}
$$

which can also be shown to apply to other hadron masses generically denoted as $M_{\mathrm{h}}$. $\mathrm{dChPT}$ thus predicts that, in the large-mass regime, the theory behaves approximately as a massdeformed conformal theory. Given the spectral results shown in Fig. 1, this is the first success of dChPT. The intuitive reason for hyperscaling is that, with $m \gg c_{1} \mathcal{M}$, the fermion mass is the dominant source of the breaking of scale invariance.

While all particle masses exhibit hyperscaling, the pion and the dilaton are still parametrically the lightest particles in the theory: $M_{\pi} \sim M_{\tau} \sim c_{1}$, which, by assumption, is a small parameter. In fact,

$$
\frac{M_{\pi}^{2}}{\left(4 \pi F_{\pi}\right)^{2}} \sim c_{1} v(m) \sim c_{1} \log \frac{m}{c_{1} \mathcal{M}},
$$

which implies that the expansion underlying dChPT is systematic as long as $c_{1} \log \frac{m}{c_{1} \mathcal{M}} \ll 1$, even though $m / \mathcal{M}$ may be large. By contrast, ordinary ChPT for QCD is only valid when $m$ is small relative to the infrared scale of the (massless) theory.

\section{Fits to LSD data [1, 2]}

One would like to test dChPT beyond the semi-quantitative observation of hyperscaling. Here we review some of the fits performed in Ref. [11] of the tree-level predictions to data for the pion mass and decay constant, as well as the dilaton mass, as a function of the fermion mass. ${ }^{1}$ The ensembles of Ref. [2] all have the same bare coupling, and fermion masses

\footnotetext{
${ }^{1}$ The dilaton decay constant was not measured in Refs. [1-3].
} 

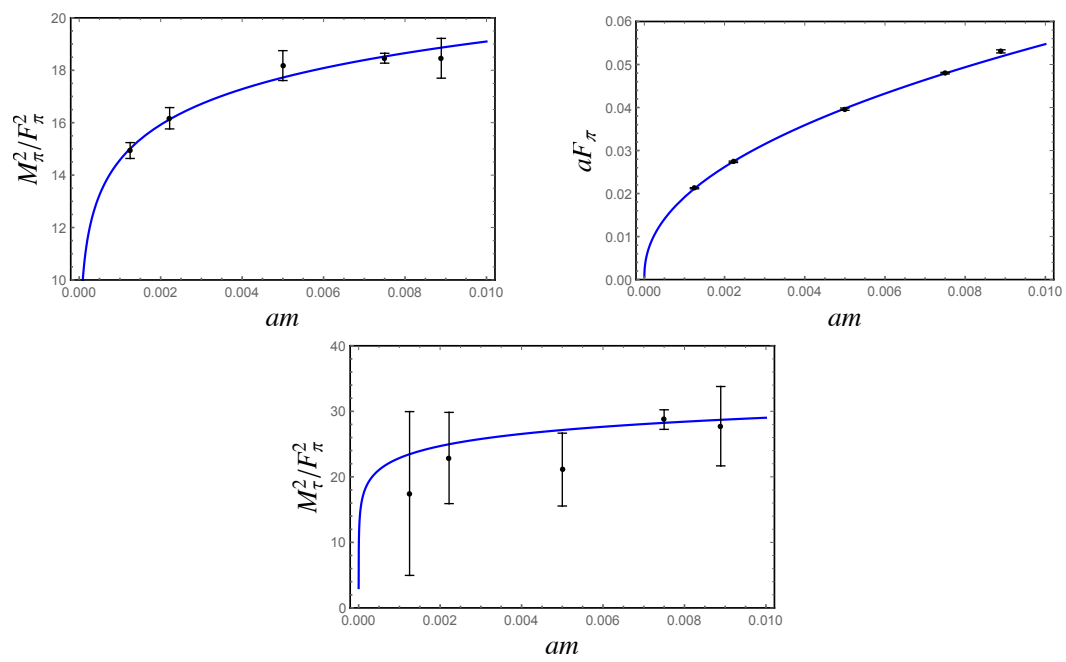

Figure 2. $M_{\pi}^{2} / F_{\pi}^{2}, a F_{\pi}$ and $M_{\tau}^{2} / F_{\pi}^{2}$ as a function of the bare fermion mass in lattice units, am. Taken from Ref. [11].

$10^{3} \mathrm{am}=(1.25,2.22,5.00,7.50,8.89)$. In Fig. 2 we show results of one of the fits of Ref. [11], using data for $M_{\pi}^{2} / F_{\pi}^{2}, a F_{\pi}$ and $M_{\tau}^{2} / F_{\pi}^{2}$ at the lowest four $a m$ values. The fit has a $p$-value of 0.89. Including also the largest mass leads to a less good fit, but the $p$-value is still 0.29 . We conclude that tree-level dChPT provides an excellent description of the LSD data. ${ }^{2}$

The parameters controlling the mass dependence are well determined by these fits: we find $\gamma_{*}=0.94(2)$ and $a B_{\pi}=2.1(1)$. In contrast, finding $a f_{\pi}$ requires a long extrapolation to the chiral limit, and the result of the fits is $a f_{\pi}=0.0006(3)$.

We emphasize that the data all have $0.02 \lesssim a F_{\pi}(m) \lesssim 0.06$, with $F_{\pi} L \gtrsim 1$ and certainly $M_{\pi} L \gg 1$, so that the data are all in the $p$-regime. However, as already follows from the approximate hyperscaling, the LSD fermion masses are solidly in the large-mass regime, hence the long extrapolation needed to reach the chiral limit. This long extrapolation may make the value of $a f_{\pi}$ very sensitive to higher orders in dChPT, even if next-to-leading order (NLO) corrections at the LSD masses are relatively small. The (tentative) conclusion is that reaching the chiral limit while keeping $f_{\pi} L \gtrsim 1$ would require unrealistically large volumes, if the same bare coupling is kept.

The LSD collaboration also measured two of the staggered pion taste splittings (specifically, the "axial" and "tensor" ones). The taste splittings look very different from those found in QCD, which are explained well by the staggered extension of ordinary ChPT. Once we extend dChPT to include the discretization effects of staggered fermions (SdChPT for short), we find that these taste splittings can be quantitatively understood using SdChPT. For details, see Ref. [11]; we will show an example of taste splittings in the next section.

\section{Fits to LatKMI data [3]}

Next, we consider fitting dChPT to the data of Ref. [3]. The LatKMI collaboration considered the same theory, with fermion masses $10^{2} \mathrm{am}=$ $(1.2,1.5,2.0,3.0,4.0,5.0,6.0,7.0,8.0,10.0)$, significantly larger than those of

\footnotetext{
${ }^{2}$ For other applications of tree-level dChPT, see Refs. [5, 6].
} 


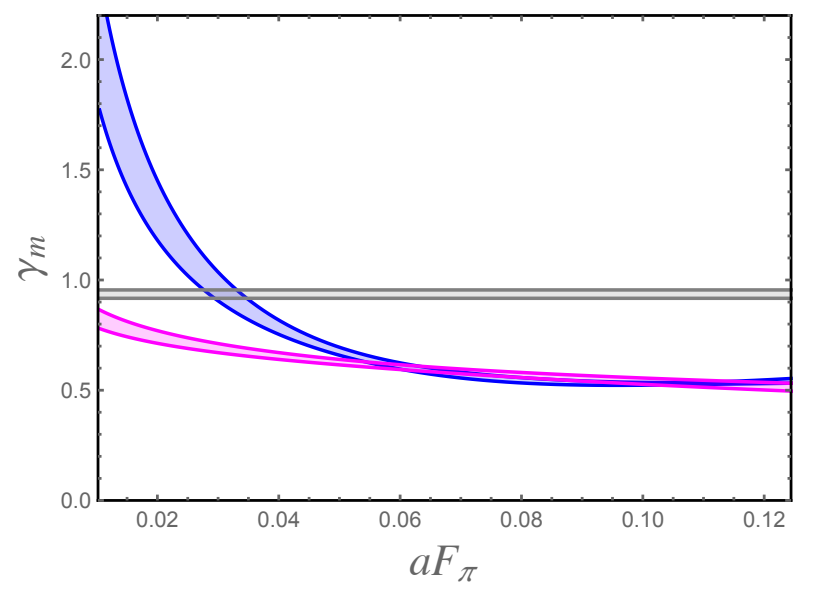

Figure 3. The running mass anomalous dimension $\gamma(m)$, obtained from various fits of the LatKMI data, as a function of $a F_{\pi}(m)$, see text. Taken from Ref. [12].

the LSD collaboration. Since the LatKMI and LSD collaborations used different lattice actions, and both collaborations did simulations at a single value of the bare coupling, we cannot compare the two data sets in physical units. In other words, we do not know the relative size of the LatKMI and LSD lattice spacings.

In Ref. [12] we found that we cannot fit the LatKMI data over their full mass range with tree-level dChPT. Fits of subsets suggest that NLO corrections will have to be taken into account. However, at NLO the effective theory has a large number of new LECs [8], and there are insufficient data to resolve these, even if the actual fits contain a smaller number of linear combinations of the new LECs.

Instead, we found that the data are well described with an $m$-dependent mass anomalous dimension $^{3}$

$$
\gamma(m)=\gamma_{0}-b v(m)+c v^{2}(m) .
$$

For a detailed description of the fits we refer to Ref. [12]. Here we just show the results we obtain for $\gamma(m)$ of Eq. (8), in Fig. 3. The blue band shows a fit $(p$-value $=0.48)$ to data at all but the largest mass of the LatKMI range, with all three parameters, $\gamma_{0}, b$ and $c$. The magenta band shows a fit ( $p$-value $=0.19$ ) with the two largest masses omitted, and $c=0$. For comparison, the gray band represents $\gamma_{*}=0.94(2)$ obtained from the LSD data. The actual data all lie in the range $0.045 \lesssim a F_{\pi} \lesssim 0.12$, where the two bands overlap, as expected. Again, the LatKMI masses are in the large-mass regime, requiring a long extrapolation to the chiral limit. These results corroborate the sensitivity of the chiral limit to higher orders, even if those higher orders can be small in the range of the data. Indeed, small corrections to $v(m)$ over a limited $m$-interval make a larger effect on the "hyperscaling factor" $e^{v(m)}$ in the tree-level expressions (4), which magnifies if $m$ is taken outside the range of the actual data.

To conclude this section, we show in Fig. 4 a fit of the taste splittings to tree-level SdChPT augmented by the varying $\gamma(m)$ of Eq. (8). ${ }^{4}$ These taste splittings behave very different from

\footnotetext{
${ }^{3}$ In Ref. [12] we proved that this choice is still consistent with the Ward-Takahashi identities for scale invariance. One can think of Eq. (8) as arising from a partial resummation of higher orders.

${ }^{4}$ Ref. [3] measured taste splittings only for a subset of seven fermion masses with a maximum value 0.08. Setting $c=0$ yields a good fit $(p$-value $=0.44)$ to tree-level SdChPT with $\gamma(m)$ of Eq. (8).
} 


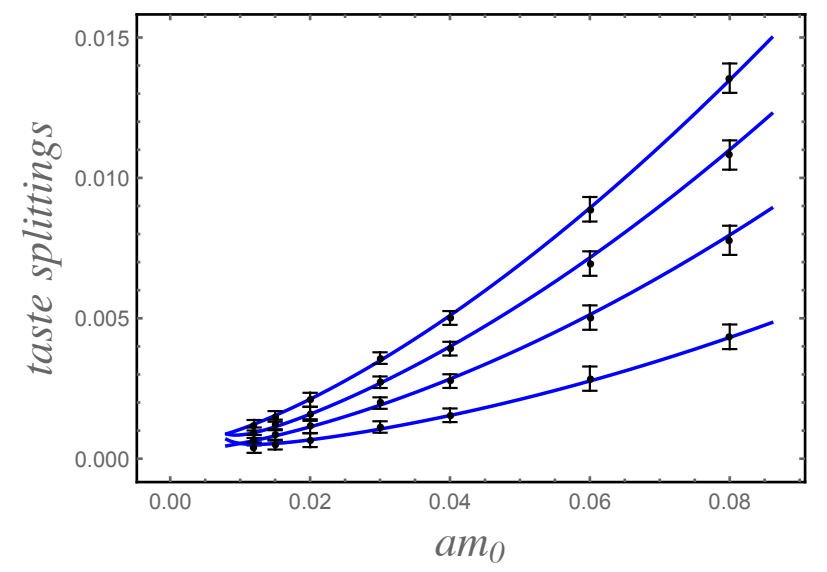

Figure 4. Fit of tree-level SdChPT to the taste splittings of Ref. [3]. Taken from Ref. [12].

those in QCD with staggered fermions, where the taste splittings are essentially independent of am. The goodness of the fit is a successful test of the dChPT framework.

\section{Conclusion}

We identified a "large-mass regime" in dChPT that has no equivalent in ordinary ChPT. This is the regime with $c_{1} \mathcal{M} \ll m \ll c_{1} \mathcal{M} e^{1 / c_{1}}$. Much like a mass-deformed infrared-conformal theory, the large-mass regime of a "walking" theory exhibits approximate hyperscaling, while the expansion underlying dChPT remains systematic, thanks to the smallness of $c_{1} \propto\left|n_{f}-n_{f}^{*}\right|$, the distance to the conformal sill. We found that current simulations of the $N_{f}=8$, SU(3) theory are deep in the large-mass regime.

Consequently, reaching the chiral limit from the data of Refs. [2, 3] requires a very long extrapolation. This extrapolation appears to be very sensitive to the mass range used in the fits, as well as to higher orders in dChPT. For example, because of this sensitivity, the chirallimit value $a f_{\pi}=0.0006(3)$ found from the LSD data may be afflicted with a larger systematic error than reflected by the statistical fit error qouted above. It is thus very difficult to determine whether $a f_{\pi}$ is actually different from zero! Likewise, it is difficult to obtain a precise value for $c_{1} \mathcal{M}$. The difficulty of controlling the long extrapolation raises the intriguing question whether, effectively, dChPT may also apply inside the conformal window, as long as the theory is mass-deformed.

Both Refs. [2] and [3] measured the dilaton mass, finding it to be nearly degenerate with the pions. But the errors are so large that the dilaton mass has little influence on the fit, as can be seen in Fig 2. Given the questions raised above it would be helpful if more precise data become available, especially at the lower fermion-mass range. In particular, this might allow for the probing of NLO corrections. Also, the size of the taste splittings indicates that scaling violations are large, and it would be nice to add another lattice spacing to study the approach to the continuum limit, about which no information is available at present.

We end with a separate comment. In Refs. [13, 14], a more general class of dilaton potentials

$$
V_{\Delta}(\tau) \propto \frac{e^{4 \tau}}{4-\Delta}\left(1-\frac{4}{\Delta} e^{(\Delta-4) \tau}\right)
$$


was considered. For $\Delta \rightarrow 4$, one recovers the dilaton potential in Eq. (2), while for $\Delta=2$ one obtains the potential for the $\sigma$-model. However, as we pointed out in Ref. [12], for values of $\Delta$ not close to 4 , there is no consistent power counting, and thus the lagrangian with this generalized potential does not constitute an effective field theory, contrary to what is claimed in Ref. [14].

\section{Acknowledgments}

MG's work is supported by the U.S. Department of Energy, Office of Science, Office of High Energy Physics, under Award Number DE-SC0013682. YS is supported by the Israel Science Foundation under grant no. 491/17.

\section{References}

[1] T. Appelquist et al. [LSD Collaboration], Lattice simulations with eight flavors of domain wall fermions in SU(3) gauge theory, Phys. Rev. D 90114502 (2014) [arXiv:1405.4752 [hep-lat]]; Strongly interacting dynamics and the search for new physics at the LHC, Phys. Rev. D 93114514 (2016) [arXiv:1601.04027 [hep-lat]].

[2] T. Appelquist et al. [Lattice Strong Dynamics], Nonperturbative investigations of SU(3) gauge theory with eight dynamical flavors, Phys. Rev. D 99014509 (2019) [arXiv:1807.08411 [hep-lat]].

[3] Y. Aoki et al. [LatKMI Collaboration], Light flavor-singlet scalars and walking signals in $N_{f}=8$ QCD on the lattice, Phys. Rev. D 96014508 (2017) [arXiv:1610.07011 [hep-lat]].

[4] Z. Fodor, K. Holland, J. Kuti, D. Nogradi, C. Schroeder and C. H. Wong, Can the nearly conformal sextet gauge model hide the Higgs impostor?, Phys. Lett. B 718, 657 (2012) [arXiv:1209.0391 [hep-lat]]; Z. Fodor, K. Holland, J. Kuti, D. Nogradi and C. H. Wong, Can a light Higgs impostor hide in composite gauge models?, PoS LATTICE 2013, 062 (2014) [arXiv:1401.2176 [hep-lat]]; The twelve-flavor $\beta$-function and dilaton tests of the sextet scalar, EPJ Web Conf. 175, 08015 (2018) [arXiv:1712.08594 [hep-lat]].

[5] Z. Fodor, K. Holland, J. Kuti and C. H. Wong, Tantalizing dilaton tests from a nearconformal EFT, PoS LATTICE 2018, 196 (2019) [arXiv:1901.06324 [hep-lat]]; Dilaton EFT from p-regime to RMT in the $\epsilon$-regime, PoS LATTICE2019, 246 (2020) arXiv:2002.05163 [hep-lat].

[6] T. Appelquist et al. [Lattice Strong Dynamics], Near-conformal dynamics in a chirally broken system, Phys. Rev. D 103014504 (2021) [arXiv:2007.01810 [hep-ph]].

[7] R. C. Brower, A. Hasenfratz, C. Rebbi, E. Weinberg and O. Witzel, Composite Higgs model at a conformal fixed point, Phys. Rev. D 93075028 (2016) [arXiv:1512.02576 [hep-ph]].

[8] M. Golterman and Y. Shamir, Low-energy effective action for pions and a dilatonic meson, Phys. Rev. D 94054502 (2016) [arXiv:1603.04575 [hep-ph]].

[9] M. Golterman and Y. Shamir, Effective field theory for pions and a dilatonic meson, PoS LATTICE 2016, 205 (2016) [arXiv:1610.01752 [hep-ph]]

[10] M. Golterman and Y. Shamir, Large-mass regime of the dilaton-pion low-energy effective theory, Phys. Rev. D 98056025 (2018) [arXiv:1805.00198 [hep-ph]].

[11] M. Golterman, E. T. Neil and Y. Shamir, Application of dilaton chiral perturbation theory to $N_{f}=8$, SU(3) spectral data, Phys. Rev. D 102034515 (2020) [arXiv:2003.00114 [hep-ph]]. 
[12] M. Golterman and Y. Shamir, Explorations beyond dilaton chiral perturbation theory in the eight-flavor SU(3) gauge theory, Phys. Rev. D 102, 114507 (2020) [arXiv:2009.13846 [hep-lat]].

[13] T. Appelquist, J. Ingoldby and M. Piai, Dilaton EFT Framework For Lattice Data, JHEP 1707, 035 (2017) [arXiv:1702.04410 [hep-ph]]; Analysis of a Dilaton EFT for Lattice Data, JHEP 1803, 039 (2018) [arXiv:1711.00067 [hep-ph]];

[14] T. Appelquist, J. Ingoldby and M. Piai, Dilaton potential and lattice data, Phys. Rev. D 101075025 (2020) [arXiv:1908.00895 [hep-ph]]. 